\title{
DAEHEE NUMBERS AND POLYNOMIALS
}

\author{
DAE SAN KIM AND TAEKYUN KIM
}

\begin{abstract}
We consider the Witt-type formula for Daehee numbrers and polynomials and investigate some properties of those numbers and polynomials. In particular, Daehee numbers are closely related to higher-order Bernoulli numbers and Bernoulli numbers of the second kind.
\end{abstract}

\section{INTRODUCTION}

As is known, the $n$-th Daehee polynomials are defined by the generating function to be

$$
\left(\frac{\log (1+t)}{t}\right)(1+t)^{x}=\sum_{n=0}^{\infty} D_{n}(x) \frac{t^{n}}{n !},(\text { see }[5,6,8,9,10,11]) .
$$

In the special case, $x=0, D_{n}=D_{n}(0)$ are called the Daehee numbers.

Throughout this paper, $\mathbb{Z}_{p}, \mathbb{Q}_{p}$ and $\mathbb{C}_{p}$ will denote the rings of $p$-adic integers, the fields of $p$-adic numbers and the completion of algebraic closure of $\mathbb{Q}_{p}$. The $p$-adic norm $|\cdot|_{p}$ is normalized by $|p|_{p}=1 / p$. Let $\operatorname{UD}\left(\mathbb{Z}_{p}\right)$ be the space of uniformly differentiable functions on $\mathbb{Z}_{p}$. For $f \in \mathrm{UD}\left(\mathbb{Z}_{p}\right)$, the $p$-adic invariant integral on $\mathbb{Z}_{p}$ is defined by

$$
I(f)=\int_{\mathbb{Z}_{p}} f(x) d \mu_{0}(x)=\lim _{n \rightarrow \infty} \frac{1}{p^{n}} \sum_{x=0}^{p^{n}-1} f(x), \text { (see [6]). }
$$

Let $f_{1}$ be the translation of $f$ with $f_{1}(x)=f(x+1)$. Then, by (1.2), we get

$$
I\left(f_{1}\right)=I(f)+f^{\prime}(0), \text { where } f^{\prime}(0)=\left.\frac{d f(x)}{d x}\right|_{x=0} .
$$

As is known, the Stirling number of the first kind is defined by

$$
(x)_{n}=x(x-1) \cdots(x-n+1)=\sum_{l=0}^{n} S_{1}(n, l) x^{l},
$$

and the Stirling number of the second kind is given by the generating function to be

$$
\left(e^{t}-1\right)^{m}=m ! \sum_{l=m}^{\infty} S_{2}(l, m) \frac{t^{l}}{l !}, \quad(\text { see }[2,3,4]) .
$$

For $\alpha \in \mathbb{Z}$, the Bernoulli polynomials of order $\alpha$ are defined by the generating function to be

$$
\left(\frac{t}{e^{t}-1}\right)^{\alpha} e^{x t}=\sum_{n=0}^{\infty} B_{n}^{(\alpha)}(x) \frac{t^{n}}{n !},(\text { see }[1,2,8]) .
$$

When $x=0, B_{n}^{(\alpha)}=B_{n}^{(\alpha)}(0)$ are called the Bernoulli numbers of order $\alpha$.

In this paper, we give a $p$-adic integral representation of Daehee numbers and polynomials, which are called the Witt-type formula for Daehee numbers and polynomials. From our integral representation, we can derive some interesting properties related to Daehee numbers and polynomials. 


\section{WitT-type formula FOR DAeheE NUmbers AND POLYNOMIALS}

First, we consider the following integral representation associated with falling factorial sequences :

$$
\int_{\mathbb{Z}_{p}}(x)_{n} d \mu_{0}(x), \text { where } n \in \mathbb{Z}_{+}=\mathbb{N} \cup\{0\} .
$$

By (2.1), we get

$$
\begin{aligned}
\sum_{n=0}^{\infty} \int_{\mathbb{Z}_{p}}(x)_{n} d \mu_{0}(x) \frac{t^{n}}{n !} & =\int_{\mathbb{Z}_{p}} \sum_{n=0}^{\infty}\left(\begin{array}{l}
x \\
n
\end{array}\right) t^{n} d \mu_{0}(x) \\
& =\int_{\mathbb{Z}_{p}}(1+t)^{x} d \mu_{0}(x),
\end{aligned}
$$

where $t \in \mathbb{C}_{p}$ with $|t|_{p}<p^{-\frac{1}{p-1}}$.

For $t \in \mathbb{C}_{p}$ with $|t|_{p}<p^{-\frac{1}{p-1}}$, let us take $f(x)=(1+t)^{x}$. Then, from (1.3), we have

$$
\int_{\mathbb{Z}_{p}}(1+t)^{x} d \mu_{0}(x)=\frac{\log (1+t)}{t} .
$$

By (1.1) and (2.3), we see that

$$
\begin{aligned}
\sum_{n=0}^{\infty} D_{n} \frac{t^{n}}{n !} & =\frac{\log (1+t)}{t} \\
& =\int_{\mathbb{Z}_{p}}(1+t)^{x} d \mu_{0}(x) \\
& =\sum_{n=0}^{\infty} \int_{\mathbb{Z}_{p}}(x)_{n} d \mu_{0}(x) \frac{t^{n}}{n !} .
\end{aligned}
$$

Therefore, by (2.4), we obtain the following theorem.

Theorem 1. For $n \geq 0$, we have

$$
\int_{\mathbb{Z}_{p}}(x)_{n} d \mu_{0}(x)=D_{n}
$$

For $n \in \mathbb{Z}$, it is known that

$$
\left(\frac{t}{\log (1+t)}\right)^{n}(1+t)^{x-1}=\sum_{k=0}^{\infty} B_{k}^{(k-n+1)}(x) \frac{t^{k}}{k !}, \quad(\text { see }[2,3,4]) .
$$

Thus, by (2.5), we get

$$
D_{k}=\int_{\mathbb{Z}_{p}}(x)_{k} d \mu_{0}(x)=B_{k}^{(k+2)}(1), \quad(k \geq 0),
$$

where $B_{k}^{(n)}(x)$ are the Bernoulli polynomials of order $n$.

In the special case, $x=0, B_{k}^{(n)}=B_{k}^{(n)}(0)$ are called the $n$-th Bernoulli numbers of order $n$.

From (2.4), we note that

$$
\begin{aligned}
(1+t)^{x} \int_{\mathbb{Z}_{p}}(1+t)^{y} d \mu_{0}(y) & =\left(\frac{\log (1+t)}{t}\right)(1+t)^{x} \\
& =\sum_{n=0}^{\infty} D_{n}(x) \frac{t^{n}}{n !}
\end{aligned}
$$


Thus, by (2.7), we get

$$
\int_{\mathbb{Z}_{p}}(x+y)_{n} d \mu_{0}(y)=D_{n}(x), \quad(n \geq 0),
$$

and, from (2.5), we have

$$
D_{n}(x)=B_{n}^{(n+2)}(x+1) .
$$

Therefore, by (2.8) and (2.9), we obtain the following theorem.

Theorem 2. For $n \geq 0$, we have

$$
D_{n}(x)=\int_{\mathbb{Z}_{p}}(x+y)_{n} d \mu_{0}(y),
$$

and

$$
D_{n}(x)=B_{n}^{(n+2)}(x+1) .
$$

By Theorem 1, we easily see that

$$
D_{n}=\sum_{l=0}^{n} S_{1}(n, l) B_{l},
$$

where $B_{l}$ are the ordinary Bernoulli numbers.

From Theorem 2, we have

$$
\begin{aligned}
D_{n}(x) & =\int_{\mathbb{Z}_{p}}(x+y)_{n} d \mu_{0}(y) \\
& =\sum_{l=0}^{n} S_{1}(n, l) B_{l}(x),
\end{aligned}
$$

where $B_{l}(x)$ are the Bernoulli polynomials defined by generating function to be

$$
\frac{t}{e^{t}-1} e^{x t}=\sum_{n=0}^{\infty} B_{n}(x) \frac{t^{n}}{n !} .
$$

Therefore, by (2.10) and (2.11), we obtain the following corollary.

Corollary 3. For $n \geq 0$, we have

$$
D_{n}(x)=\sum_{l=0}^{n} S_{1}(n, l) B_{l}(x) .
$$

In (2.4), we have

$$
\begin{aligned}
\frac{t}{e^{t}-1} & =\sum_{n=0}^{\infty} D_{n} \frac{1}{n !}\left(e^{t}-1\right)^{n} \\
& =\sum_{n=0}^{\infty} D_{n} \frac{1}{n !} n ! \sum_{m=n}^{\infty} S_{2}(m, n) \frac{t^{m}}{m !} \\
& =\sum_{m=0}^{\infty}\left(\sum_{n=0}^{m} D_{n} S_{2}(m, n)\right) \frac{t^{m}}{m !}
\end{aligned}
$$

and

$$
\frac{t}{e^{t}-1}=\sum_{m=0}^{\infty} B_{m} \frac{t^{m}}{m !} .
$$

Therefore, by (2.12) and (2.13), we obtain the following theorem. 
Theorem 4. For $m \geq 0$, we have

$$
B_{m}=\sum_{n=0}^{m} D_{n} S_{2}(m, n) .
$$

In particular,

$$
\int_{\mathbb{Z}_{p}} x^{m} d \mu_{0}(x)=\sum_{n=0}^{m} D_{n} S_{2}(m, n) .
$$

Remark. For $m \geq 0$, by (2.11), we have

$$
\int_{\mathbb{Z}_{p}}(x+y)^{m} d \mu_{0}(y)=\sum_{n=0}^{m} D_{n}(x) S_{2}(m, n) .
$$

For $n \in \mathbb{Z}_{\geq 0}$, the rising factorial sequence is defined by

$$
x^{(n)}=x(x+1) \cdots(x+n-1) .
$$

Let us define the Daehee numbers of the second kind as follows :

$$
\widehat{D}_{n}=\int_{\mathbb{Z}_{p}}(-x)_{n} d \mu_{0}(x),\left(n \in \mathbb{Z}_{\geq 0}\right) .
$$

By (2.15), we get

$$
x^{(n)}=(-1)^{n}(-x)_{n}=\sum_{l=0}^{n} S_{1}(n, l)(-1)^{n-l} x^{l} .
$$

From (2.15) and (2.16), we have

$$
\begin{aligned}
\widehat{D}_{n} & =\int_{\mathbb{Z}_{p}}(-x)_{n} d \mu_{0}(x)=\int_{\mathbb{Z}_{p}} x^{(n)}(-1)^{n} d \mu_{0}(x) \\
& =\sum_{l=0}^{n} S_{1}(n, l)(-1)^{l} B_{l} .
\end{aligned}
$$

Therefore, by (2.17), we obtain the following theorem.

Theorem 5. For $n \geq 0$, we have

$$
\widehat{D}_{n}=\sum_{l=0}^{n} S_{1}(n, l)(-1)^{l} B_{l} .
$$

Let us consider the generating function of the Daehee numbers of the second kind as follows :

$$
\begin{aligned}
\sum_{n=0}^{\infty} \widehat{D}_{n} \frac{t^{n}}{n !} & =\sum_{n=0}^{\infty} \int_{\mathbb{Z}_{p}}(-x)_{n} d \mu_{0}(x) \frac{t^{n}}{n !} \\
& =\int_{\mathbb{Z}_{p}} \sum_{n=0}^{\infty}\left(\begin{array}{c}
-x \\
n
\end{array}\right) t^{n} d \mu_{0}(x) \\
& =\int_{\mathbb{Z}_{p}}(1+t)^{-x} d \mu_{0}(x) .
\end{aligned}
$$

From (1.3), we can derive the following equation :

$$
\int_{\mathbb{Z}_{p}}(1+t)^{-x} d \mu_{0}(x)=\frac{(1+t) \log (1+t)}{t},
$$

where $|t|_{p}<p^{-\frac{1}{p}}$. 
By (2.18) and (2.19), we get

$$
\begin{aligned}
\frac{1}{t}(1+t) \log (1+t) & =\int_{\mathbb{Z}_{p}}(1+t)^{-x} d \mu_{0}(x) \\
& =\sum_{n=0}^{\infty} \widehat{D}_{n} \frac{t^{n}}{n !} .
\end{aligned}
$$

Let us consider the Daehee polynomials of the second kind as follows :

$$
\frac{(1+t) \log (1+t)}{t} \frac{1}{(1+t)^{x}}=\sum_{n=0}^{\infty} \widehat{D}_{n}(x) \frac{t^{n}}{n !} .
$$

Then, by (2.21), we get

$$
\int_{\mathbb{Z}_{p}}(1+t)^{-x-y} d \mu_{0}(y)=\sum_{n=0}^{\infty} \hat{D}_{n}(x) \frac{t^{n}}{n !} .
$$

From (2.22), we get

$$
\begin{aligned}
\widehat{D}_{n}(x) & =\int_{\mathbb{Z}_{p}}(-x-y)_{n} d \mu_{0}(y), \quad(n \geq 0) \\
& =\sum_{l=0}^{n}(-1)^{l} S_{1}(n, l) B_{l}(x) .
\end{aligned}
$$

Therefore, by (2.23), we obtain the following theorem.

Theorem 6. For $n \geq 0$, we have

$$
\widehat{D}_{n}(x)=\int_{\mathbb{Z}_{p}}(-x-y)_{n} d \mu_{0}(y)=\sum_{l=0}^{n}(-1)^{l} S_{1}(n, l) B_{l}(x) .
$$

From (2.21) and (2.22), we have

$$
\begin{aligned}
\left(\frac{t}{e^{t}-1}\right) e^{(1-x) t} & =\sum_{n=0}^{\infty} \widehat{D}_{n}(x) \frac{1}{n !}\left(e^{t}-1\right)^{n} \\
& =\sum_{n=0}^{\infty} \widehat{D}_{n}(x) \frac{1}{n !} n ! \sum_{m=n}^{\infty} S_{2}(m, n) \frac{t^{m}}{m !} \\
& =\sum_{m=0}^{\infty}\left(\sum_{n=0}^{m} \widehat{D}_{n}(x) S_{2}(m, n)\right) \frac{t^{n}}{m !}
\end{aligned}
$$

and

$$
\begin{aligned}
\int_{\mathbb{Z}_{p}} e^{-(x+y) t} d \mu_{0}(y) & =\sum_{n=0}^{\infty} \widehat{D}_{n}(x) \frac{\left(e^{t}-1\right)^{n}}{n !} \\
& =\sum_{m=0}^{\infty}\left(\sum_{n=0}^{m} \widehat{D}_{n}(x) S_{2}(m, n)\right) \frac{t^{m}}{m !}
\end{aligned}
$$

Therefore, by (2.24) and (2.25), we obtain the follwoing theorem.

Theorem 7. For $m \geq 0$, we have

$$
\begin{aligned}
B_{m}(1-x) & =(-1)^{m} \int_{\mathbb{Z}_{p}}(x+y)^{m} d \mu_{0}(y) \\
& =\sum_{n=0}^{m} \widehat{D}_{n}(x) S_{2}(m, n) .
\end{aligned}
$$


In particular,

$$
B_{m}(1-x)=(-1)^{m} B_{m}(x)=\sum_{n=0}^{m} \widehat{D}_{m}(x) S_{2}(m, n) .
$$

Remark. By (2.5), (2.20) and (2.21), we see that

$$
\widehat{D}_{n}=B_{n}^{(n+2)}(2), \quad \widehat{D}_{n}(x)=B_{n}^{(n+2)}(2-x) .
$$

From Theorem 1 and (2.15), we have

$$
\begin{aligned}
(-1)^{n} \frac{D_{n}}{n !} & =(-1)^{n} \int_{\mathbb{Z}_{p}}\left(\begin{array}{l}
x \\
n
\end{array}\right) d \mu_{0}(x) \\
& =\int_{\mathbb{Z}_{p}}\left(\begin{array}{c}
-x+n-1 \\
n
\end{array}\right) d \mu_{0}(x) \\
& =\sum_{m=0}^{n}\left(\begin{array}{c}
n-1 \\
n-m
\end{array}\right) \int_{\mathbb{Z}_{p}}\left(\begin{array}{c}
-x \\
m
\end{array}\right) d \mu_{0}(x) \\
& =\sum_{m=0}^{n}\left(\begin{array}{c}
n-1 \\
n-m
\end{array}\right) \frac{\widehat{D}_{m}}{m !}=\sum_{m=1}^{n}\left(\begin{array}{c}
n-1 \\
m-1
\end{array}\right) \frac{\widehat{D}_{m}}{m !},
\end{aligned}
$$

and

$$
\begin{aligned}
(-1)^{n} \frac{\widehat{D}_{n}}{n !} & =(-1)^{n} \int_{\mathbb{Z}_{p}}\left(\begin{array}{c}
-x \\
n
\end{array}\right) d \mu_{0}(x)=\int_{\mathbb{Z}_{p}}\left(\begin{array}{c}
x+n-1 \\
n
\end{array}\right) d \mu_{0}(x) \\
& =\sum_{m=0}^{n}\left(\begin{array}{c}
n-1 \\
n-m
\end{array}\right) \int_{0}^{1}\left(\begin{array}{c}
x \\
m
\end{array}\right) d \mu_{0}(x) \\
& =\sum_{m=0}^{n}\left(\begin{array}{c}
n-1 \\
m-1
\end{array}\right) \frac{D_{m}}{m !}=\sum_{m=1}^{n}\left(\begin{array}{c}
n-1 \\
m-1
\end{array}\right) \frac{D_{m}}{m !}
\end{aligned}
$$

Therefore, by (2.26) and (2.27), we obtain the following theorem.

Theorem 8. For $n \in \mathbb{N}$, we have

$$
(-1)^{n} \frac{D_{n}}{n !}=\sum_{m=1}^{n}\left(\begin{array}{c}
n-1 \\
m-1
\end{array}\right) \frac{\widehat{D}_{m}}{m !}
$$

and

$$
(-1)^{n} \frac{\widehat{D}_{n}}{n !}=\sum_{m=1}^{n}\left(\begin{array}{c}
n-1 \\
m-1
\end{array}\right) \frac{D_{m}}{m !}
$$

\section{REFERENCES}

[1] A. Bayad, Special values of Lerch zeta function and their Fourier expansions, Adv. Stud. Contemp. Math. 21 (2011), no. 1, 1-4.

[2] L. Carlitz, A note on Bernoulli and Euler polynomials of the second kind, Scripta Math. 25 (1961), 323-330.

[3] L. Comtet, Advanced Combinatorics, Reidel, Dordrecht, 1974.

[4] H. W. Gould, Explicit formulas for Bernoulli numbers, Amer. Math. Monthly 79(1972), 4451.

[5] T. Kim, D. S. Kim, T. Mansour, S. H. Rim, M. Schork, Umbral calculus and Sheffer sequence of polynomials, J. Math. Phys. 54, 083504 (2013) : http://dx.doi.org/10.1063/1.4817853 (15 pages)

[6] T. Kim, An invariant p-adic integral associated with Daehee numbers, Integral Transforms Spec. Funct. 13 (2002), no. 1, 65-69.

[7] V. Kurt, Some relation between the Bernstein polynomials and second kind Bernoulli polynomials, Adv. Stud. Contemp. Math. 23 (2013), no. 1, 43-48.

[8] H. Ozden, I. N. Cangul, Y. Simsek, Remarks on q-Bernoulli numbers associated with Daehee numbers, Adv. Stud. Contemp. Math (Kyungshang) 18 (2009), no. 1, 41-48. 
[9] Y. Simsek, S-H. Rim, L. -C. Jang, D.-J. Kang, J.-J. Seo, A note q-Daehee sums, Proceedings of the 16th International Conference of the Jangjeon Mathematical Society, 159-166, Jangjeon Math. Soc., Hapcheon, 2005.

[10] Y. Simsek, Special functions related to Dedekind-type DC-sums and their applications, Russ. J. Math. Phys. 17 (2010), no. 4, 495-508

Department of Mathematics, Sogang University, Seoul 121-742, Republic of Korea e-mail:dskim@sogang.ac.kr

Department of Mathematics, Kwangwoon University, Seoul 139-701, Republic of Korea

e-mail:tkkim@kw.ac.kr 